\title{
Dynamic merge of discrete goods flow - Impact on throughput and efficiency
}

\author{
Simon Gasperin \\ Dirk Jodin \\ Institute of Logistics Engineering \\ Graz University of Technology, Austria
}

C ontinuous conveyors with a dynamic merge were developed with adaptable control equipment to differentiate these merges from competing Stop-and-Go merges. With a dynamic merge, the partial flows are manipulated by influencing speeds so that transport units need not stop for the merge. This leads to a more uniform flow of materials, which is qualitatively observable and verifiable in long-term measurements. And although this type of merge is visually mesmerizing, does it lead to advantages from the view of material flow technology? Our study with real data indicates that a dynamic merge shows a $24 \%$ increase in performance, but only for symmetric or nearly symmetric flows. This performance advantage decreases as the flows become less symmetric, approaching the throughput of traditional Stop-and-Go merges. And with a cost premium for a continuous merge of approximately $10 \%$ due to the additional technical components (belt conveyor, adjustable drive engines, software, etc.), this restricts their economical use.

[Keywords: dynamik merge, throughput, material flow calculation, efficiency, throughput diagram]

\section{INTRODUCTION}

Sustainability has attained a high meaning also in the distribution and production logistics. In the future the goals of technical arrangements of Intralogistics are no more only productive and economic efficiency, but also flexibility, adaptiveness, energy- and resource efficiency become important competitive criterias. It is obvious that the replacement of electric motors against more energy saving motors is insufficient. There are more factors in many areas like mechanics, the control system and the operating state itself with possible savings which have to be considered in the overall view. Only the intelligent combination of appropriate actions will be able to fulfill the above mentioned criteria to fit together harmoniously. Summarized this means that activity for energy efficiency or resource conservation are not allowed to influence the functionality and economic efficiency. For making these decisions there must be a wide, competent knowledge of all the important influencing factors. In the range of a research project supported by Österreichische Forschungs- förderungsgesellschaft FFG (Austrian research support society), the institute of logistics engineering at Graz University of Technology and an industrial partner will work out together such fundamentals, for developing properties, measuring methods and implementations within the next three years. At the same time the different possibilities of technology, the control system and operating strategy should be put to each other in relation to compare them with each other. In connection with an improved company strategy for material flow systems, the potentials of a dynamic merge of steady material flows compared to traditional Stop-and-Go procedures are analyzed. The result of the investigation will be the topic of the following article.

First of all the basics of material flow of common Stop-and-Go merges and prioritized merges will be discussed, so afterwards in chapter three it is possible to educe the principles of a dynamic merge. In chapter four the technical boundary of this solution is discussed and in the next chapter the potentials for the practice will be estimated with the help of arithmetic examples, which have real numerical values.

\section{Merging Protocols}

Merges are found in many areas of material handling facilities. They are used in the receiving, in the supply of sorter systems, in the transfer area of high bay racks or in the consignment. With respect to their rights of way, merges of two streams (see illustration 1) are classified into "first come, first served" (FCFS) merges and prioritized merges.

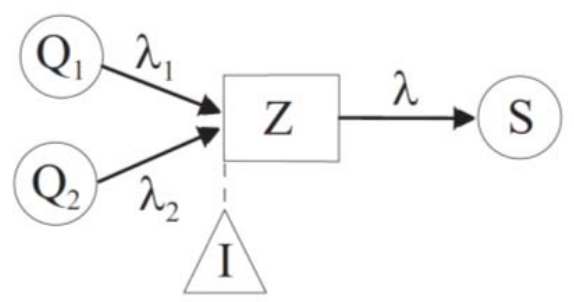

Illustration 1. System sketch of the Stop-and-Go merge (compare [ArF05]). 
A prioritization gets developed if the main flow must not be influenced by the merge and the objects in the sideflow wait as long as the merge is possible without an obstruction. For e.g.: highway entrances in the traffic or merges on the distribution conveyor with sorter. Here dynamic prioritized merges are natural. With the help of speed adaptations it is tried to merge the object into a gap of the main flow at very high speed to reach a high throughput. Only if no suitable gap originates, the object is stopped [Jod10].

For equal merges it is usual to use stoppers, which mutually interrupt the partial flows to guarantee a regulated merge. The system is sketched in illustration 1 for two partial flows.

Based on the sources $Q_{1}$ and $Q_{2}$ the material flow with the throughputs $\lambda_{1}$ and $\lambda_{2}$ are brought together in $\mathrm{Z}$ according to FCFS. Which of the objects has right of way is decided at checkpoint I. Here the rule FCFS counts. The change causes switch losses by the response times and acceleration times of the actuating elements in the range of $0.1 \mathrm{sec}$. Times of braking and acceleration have to be added. They are dependent of the conveyor speeds and also of the throughput. ARNOLD and FURMANS deliver a mathematical view of the Stop-and-Go technology by using inequalities [ArF05], which are based on the investigation of GUDEHUS [Gud76]. From the ,general limiting performance law", the following inequality is put up for the Stop and Go merge of two part flows:

$$
\frac{\lambda_{1}}{\mu_{1}}+\frac{\lambda_{2}}{\mu_{2}}+\omega \cdot t_{\mathrm{S}} \leq 1
$$

On this occasion, the maximum throughputs of the merged flows are explained by $\mu_{1}$ and $\mu_{2}$. The size $\omega$ defines the switch frequency in the merge and $t_{S}$ the switch time. The switch frequency $\omega$ for two partial flows is defined by the following equation:

$$
\omega=2 \cdot \frac{\lambda_{1} \cdot \lambda_{2}}{\lambda_{1}+\lambda_{2}}
$$

By inserting (2) into (1) the entire inequality of the Stopand-Go merge is summarized as,

$$
\frac{\lambda_{1}}{\mu_{1}}+\frac{\lambda_{2}}{\mu_{2}}+2 \cdot \frac{\lambda_{1} \cdot \lambda_{2}}{\lambda_{1}+\lambda_{2}} \cdot \mathrm{t}_{\mathrm{S}} \leq 1
$$

The boundary of this inequality can be qualitatively shown in the so-called flow diagram (see illustration 2).

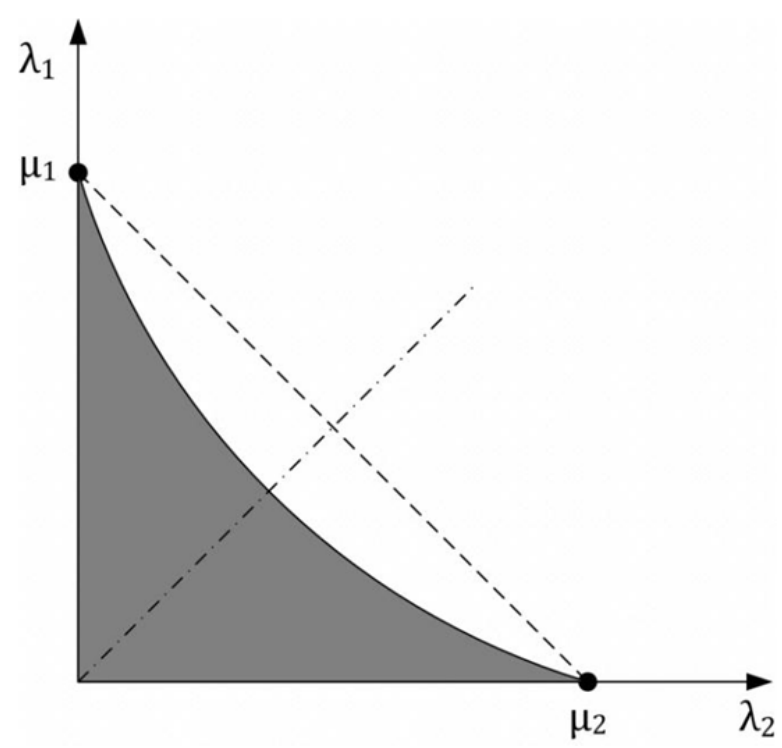

Illustration 2. Throughput diagram of the Stop-and-Go strategy.

This diagram shows all the space for solutions of the inequality. The shaded surface defines the valid value area within all possible combinations $\left(\lambda_{1}, \lambda_{2}\right)$ of the merge that can be passed without a traffic jam. The connection line between $\mu_{1}$ and $\mu_{2}$ shows the solution without switching time portion. That's why it is seen as an improved theoretical boundary. Underneath there is a curved connection line which shows the loss caused by switching time. The performance decreasing effect of the switching time is clearly recognizable by the distance between the lines, which reaches the maximum by symmetrical load.

In view of rising efficiency requirements for material flow systems also in case of equal merges, actions have to be discussed and partially realized, to increase the throughput and the total efficiency. The distances and speeds of the units should be influenced by an additional deceleration- acceleration unit in every partial flow before the merge. As a result all units stay in movement and no stop becomes necessary.

\section{DYNAMiC MERge}

Although such merges are mesmerizing in their dynamic behavior, quantify the expected improvements of a dynamic merge is important. Illustration 1 can be extended with the brake - and acceleration units (BBE) as shown in illustration 3. 


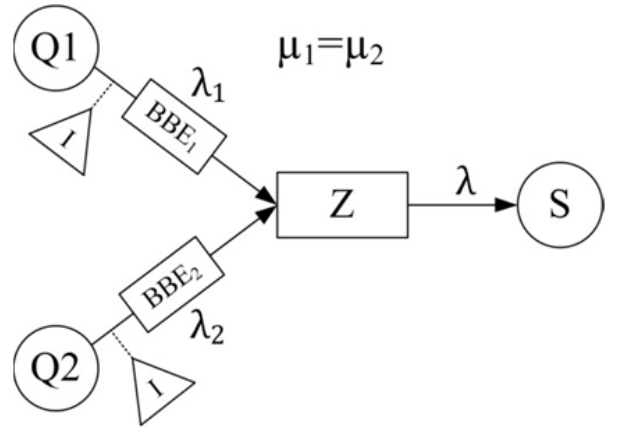

Illustration 3. System sketch of the dynamic merge.

Switching time $t_{S}$ from (3) is substituted by a time for distance building. To describe this system mathematically, discrete elements must be defined for the material flow. The special case of constant gaps between the transport units (TU) is observed (see Quasi-stationary look on the system).

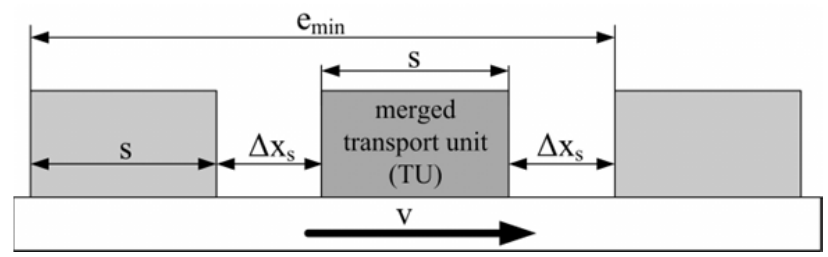

Illustration 4. Quasi-stationary look on the system.

Table 1. Possibilities of the dynamic merge
Provided that conveyor speed $\mathrm{v}$ is constant, a change of the throughput $\lambda$ can only be caused by a variation of the distance e. Distance e contains the object length s and the favored safety distance $\Delta x_{s}$. Furthermore a symmetrical merge is assumed. Therefore the system is a connection knot of two partial flows with same length ( $\mathrm{s}=$ constant) and maximum throughputs $\left(\mu_{1}=\mu_{2}\right)$. The distance e can be derived from the conveyor speed $\mathrm{v}$ and the throughput $\lambda$.

$$
\lambda=\frac{v}{e} \Rightarrow e=\frac{v}{\lambda}
$$

So it is necessary to clarify the issue in which situations a braking and acceleration must be initiated. For the merge of a transport unit of a partial flow, the gap in the other partial flow must be at least the object length $\mathrm{s}$ plus the minimum distances $2 \Delta x_{s}$. The necessary minimum distance $e_{\text {min }}$ between two units into whose gap should be merged, is $e_{\min }=2 \cdot s+2 \cdot \Delta x_{s}$. If there is a smaller distance it must be intervened kinematically. The throughput $\lambda_{k}$ which must not be limited yet calculates itself with:

$$
\lambda_{k}=\frac{v}{e_{\min }}=\frac{v}{2 \cdot s+2 \cdot \Delta x_{s}}
$$

Table 1 shows the four value areas that yield with the merge of two partial streams. The quantities $a_{1}$ and $a_{2}$ define the accelerations and decelerations in the conveyor distances one and two.

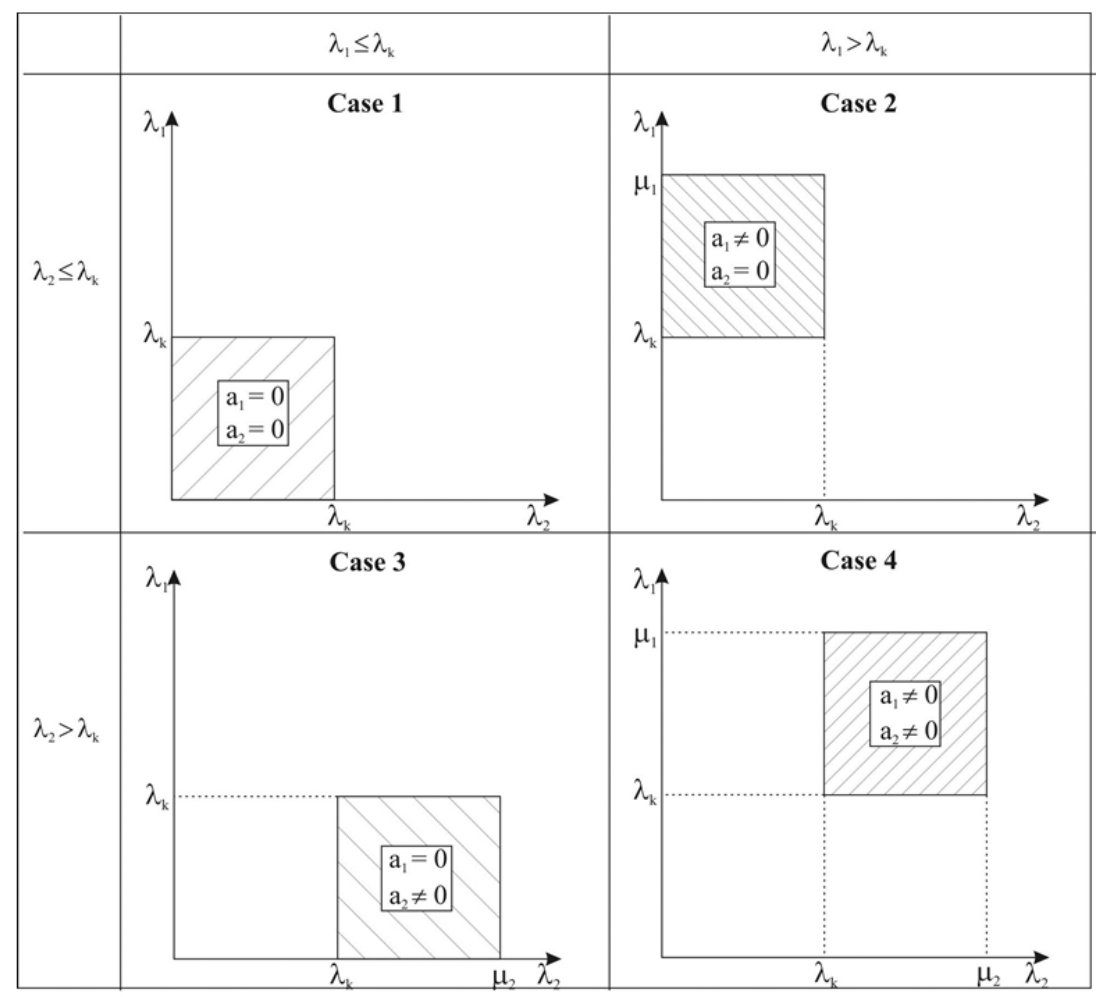


If the allowed combinations from $\lambda_{1}$ and $\lambda_{2}$ are marked into a common throughput diagram, maximum validity areas originate.

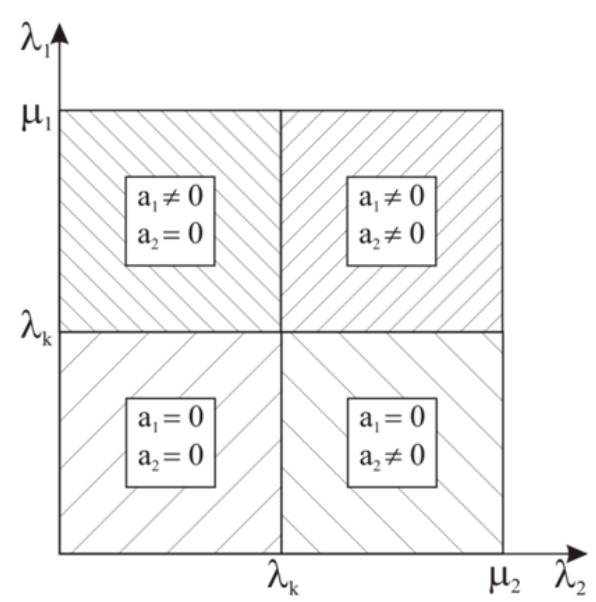

Illustration 5. Validity areas in the throughput diagram

Now there is a need to search a mathematical formulation for each of these four cases. For the simplification the deceleration function is defined as an infinite positive or negative acceleration. The necessary gap should be always generated in the same size by acceleration of the front object and the simultaneous deceleration of the following object. In illustration 6 the procedure is shown, where attention should be paid to, that the illustration's left and right parts happen at the same time.

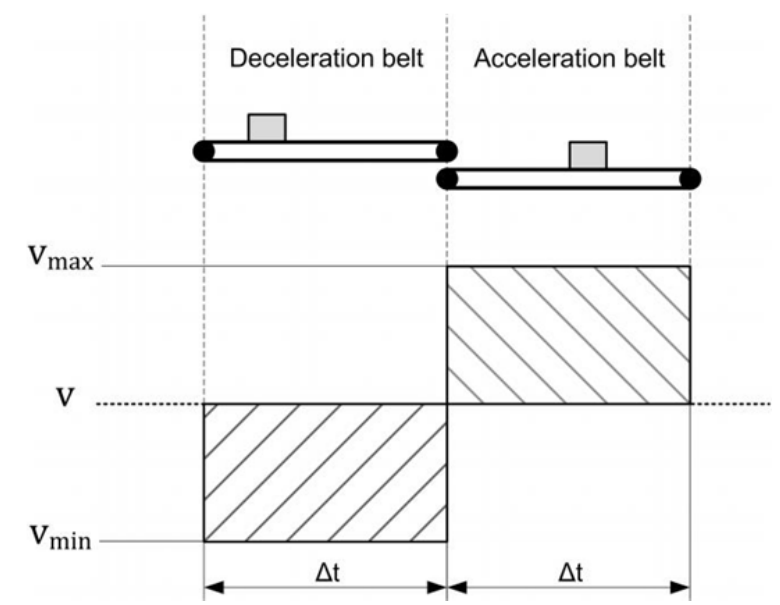

Illustration 6. Validity areas in the throughput diagram.

The acceleration time is $\Delta \mathrm{t}$, the conveyor speed is $\mathrm{v}$.
The distance enlargement as a function of the time results from the speed difference:

$$
\Delta \mathrm{s}=\left(\mathrm{v}_{\max }-\mathrm{v}_{\min }\right) \cdot \Delta \mathrm{t}=\Delta \mathrm{v} \cdot \Delta \mathrm{t}
$$

The necessary distance enlargement is calculated from throughput, conveyor speed and minimal distance:

$$
\Delta \mathrm{s}=\mathrm{e}_{\min }-\mathrm{e}_{\mathrm{ist}}=\left(2 \cdot \mathrm{s}+2 \cdot \Delta \mathrm{x}_{\mathrm{s}}\right)-\frac{\mathrm{v}}{\lambda}
$$

The necessary deceleration- acceleration time arises by equating (6) and (7) and solving for $\Delta \mathrm{t}$ :

$$
\Delta \mathrm{t}=\frac{2 \cdot \mathrm{s}+2 \cdot \Delta \mathrm{x}_{\mathrm{s}}-\frac{\mathrm{v}}{\lambda}}{\left(\mathrm{v}_{\max }-\mathrm{v}_{\min }\right)}
$$

This time for distance formation substitutes the switching time for the Stop-and-Go merge. However it is not constant but a function of several variables. The inequalities of the dynamic merges consist of up to three components.

1. Sum of the usage rates: $\frac{\lambda_{1}}{\mu_{1}}+\frac{\lambda_{2}}{\mu_{2}}$

2. Control frequency: $\quad \omega=\mathrm{f}\left(\lambda_{1}, \lambda_{2}\right)$

This component considers the necessary correction quantity of the gap length by deceleration and acceleration processes. According to illustration 5 four cases arise:
1. $\mathrm{a}_{1}=0 ; \mathrm{a}_{2}=0 \Rightarrow \omega=0$
2. $\mathrm{a}_{1} \neq 0 ; \mathrm{a}_{2}=0 \Rightarrow \omega=\lambda_{1}$
3. $\mathrm{a}_{1}=0 ; \mathrm{a}_{2} \neq 0 \Rightarrow \omega=\lambda_{2}$
4. $\mathrm{a}_{1} \neq 0 ; \mathrm{a}_{2} \neq 0 \quad \Rightarrow \quad \omega_{1}=\lambda_{1} ; \omega_{2}=\lambda_{2}$

3. Control time: $\quad \mathrm{t}=\mathrm{f}\left(\lambda_{1} ; \lambda_{2} ; \mathrm{s} ; \omega ; \Delta \mathrm{v} ; \Delta \mathrm{x}_{\mathrm{S}}\right)$

The necessary time for every control process, which is a function of the distance between the transport units of the respectively other partial flow $\mathrm{f}\left(\lambda_{1}, \lambda_{2}\right)$ the length of the transport units $\mathrm{s}$, the conveyor speed $\mathrm{v}$, the technically possible speed difference $\Delta v$ and the required safe distance $\Delta x_{S}$ between the transport units.

Here from it is possible to put up the inequalities for the four different cases from table 1 , as shown in table 2. 
Table 2. inequalities for the four different cases

\begin{tabular}{|c|l|}
\hline no & inequalities \\
\hline 1 & $\frac{\lambda_{1}}{\mu_{1}}+\frac{\lambda_{2}}{\mu_{2}} \leq 1$ \\
\hline 2 & $\frac{\lambda_{1}}{\mu_{1}}+\frac{\lambda_{2}}{\mu_{2}}+\lambda_{1} \cdot \frac{2 \cdot \mathrm{s}+2 \cdot \Delta \mathrm{x}_{\mathrm{s}}-\frac{\mathrm{v}}{\lambda_{2}}}{\Delta \mathrm{v}} \leq 1$ \\
\hline 3 & $\frac{\lambda_{1}}{\mu_{1}}+\frac{\lambda_{2}}{\mu_{2}}+\lambda_{2} \cdot \frac{2 \cdot \mathrm{s}+2 \cdot \Delta \mathrm{x}_{\mathrm{s}}-\frac{\mathrm{v}}{\lambda_{1}}}{\Delta \mathrm{v}} \leq 1$ \\
\hline 4 & $\frac{\lambda_{1}}{\mu_{1}}+\frac{\lambda_{2}}{\mu_{2}}+\lambda_{1} \cdot \frac{2 \cdot \mathrm{s}+2 \cdot \Delta \mathrm{x}_{\mathrm{s}}-\frac{\mathrm{v}}{\lambda_{2}}}{\Delta \mathrm{v}}+\lambda_{2} \cdot \frac{2 \cdot \mathrm{s}+2 \cdot \Delta \mathrm{x}_{\mathrm{s}}-\frac{\mathrm{v}}{\lambda_{1}}}{\Delta \mathrm{v}} \leq 1$ \\
\hline
\end{tabular}

The functions can also be shown in the throughput diagram. With the basis of the curves, qualitatively shown in illustration 7 , the effects of a dynamic prioritized merge can be discussed.

The boundary of the maximum throughput is marked by the straight connection of the maximum throughputs $\mu_{1}$ and $\mu_{2}$, without buildups and switch losses. The curves of the inequalities (2)-(4) of Table 2 are shown dotted beyond their respective validity area. The validity areas of the three curves have common intersections and also connect the maximum throughputs on the axes of co- ordinates. The series of curves shows the maximum throughput of the dynamic merge. The shown sketched line represents the maximum throughput of the classical Stop-and-Go merge.

Now we discuss the course of the series of curves from the top on the left, which begins with a maximum throughput $\mu_{1}$ of the partial flow 1, to the right below. Although partial flow 2 increases first, it is still so low that the middle distances of the TU show enough big gaps for the TU of partial flow 1, completely without deceleration. Simply partial flow 1 must be decelerated for a merge.

In the intersection with the perpendicular of the maximum throughput $\lambda_{k}$, the gaps in partial flow 2 also become too small, so that there must be also deceleration. In the intersection with the horizontals of the maximum throughput, partial flow 1 has sunk so far that enough big gaps appear without deceleration. So, only partial flow 2 needs to be decelerated. The curve ends with a maximum throughput $\mu_{2}$ of partial flow 2 on the $\mathrm{x}$-axis.

The potential of the dynamic junction arises from the horizontal and vertical distances between the dotted curve and the just discussed curve shape.

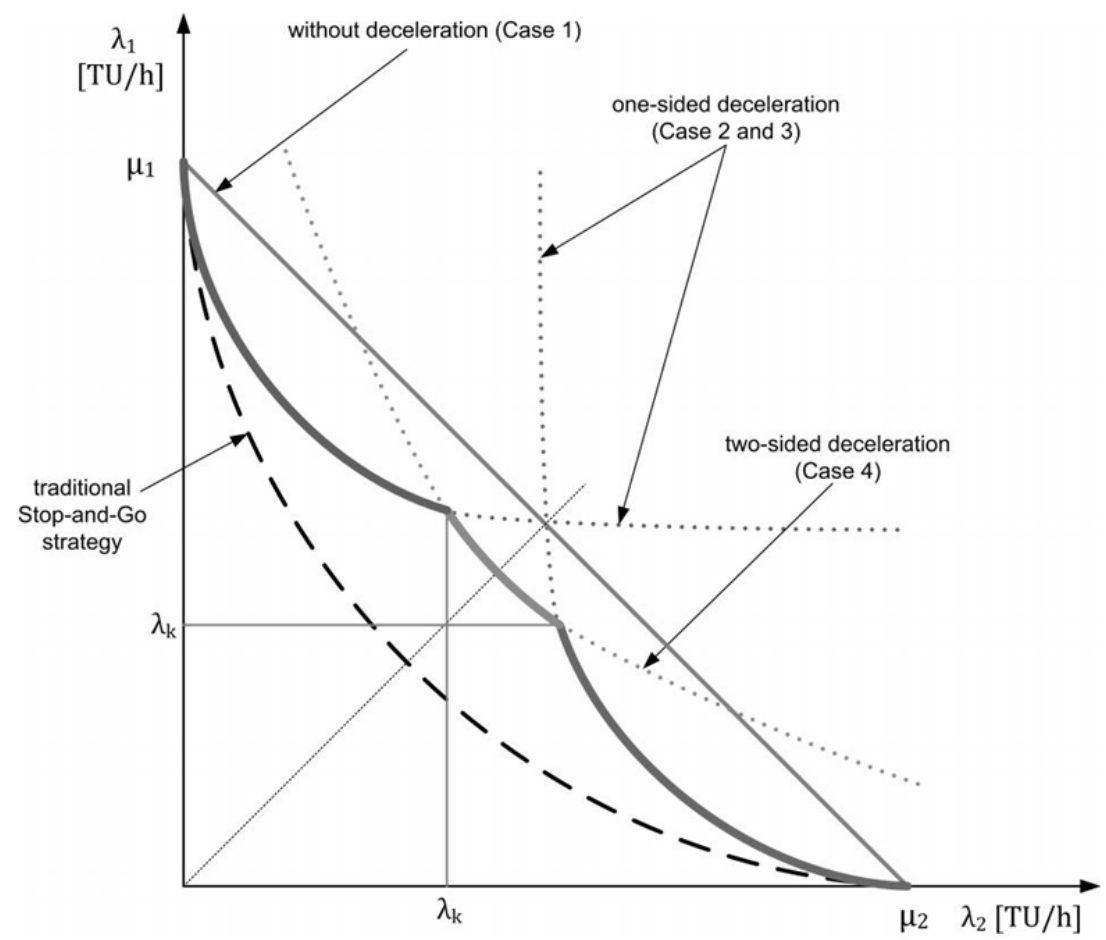

Illustration 7. Solutions of the inequalities and their accompanying fields of validity. 


\section{TECHNICAL BOUNDARY OF THE DYNAMIC MERGE}

The technical boundary of the dynamic prioritized merge defines itself by the reduction of the distance between the braked unit and the following unit. As soon as the minimal distance is reached or if the units touch each other, no more braking is possible. This state defines the technical maximum of the dynamic merge.

The caused change of the gap width $\Delta \mathrm{s}_{\text {decelerate }}$ to the following transport unit by decelerating equals half of the distance enlargement (using (6)), as shown below:

$$
\Delta \mathrm{s}_{\text {decelerate }}=\frac{\Delta \mathrm{v} \cdot \Delta \mathrm{t}}{2}=\frac{2 \cdot \mathrm{s}+2 \cdot \Delta \mathrm{x}_{\mathrm{s}}-\frac{\mathrm{v}}{\lambda}}{2} .
$$

The distance between the decelerating transport unity and the following TU may not fall below the safety distance $(\Delta \mathrm{x})$. Thereby follows the relation:

$$
\frac{\mathrm{v}}{\lambda}-\frac{2 \cdot \mathrm{s}+2 \cdot \Delta \mathrm{x}_{\mathrm{s}}-\frac{\mathrm{v}}{\lambda}}{2} \geq \mathrm{s}+\Delta \mathrm{x}_{\mathrm{s}}
$$

From equation (10), the result follows by reorganizing:

$$
\frac{3 \mathrm{v}}{2 \lambda} \geq 2 \cdot\left(\mathrm{s}+\Delta \mathrm{x}_{\mathrm{s}}\right), \text { bzw. } \frac{\mathrm{v}}{\lambda} \geq \frac{4}{3} \cdot\left(\mathrm{s}+\Delta \mathrm{x}_{\mathrm{s}}\right)
$$

Solving for $\lambda$ in (11) yields:

$$
\lambda \leq \frac{3}{4} \cdot \frac{\mathrm{v}}{\left(\mathrm{s}+\Delta \mathrm{x}_{\mathrm{s}}\right)}
$$

Therefore the technical maximum of the dynamic prioritized merge is located in the possible operating fields. So these boundaries are inserted in the throughput diagram.

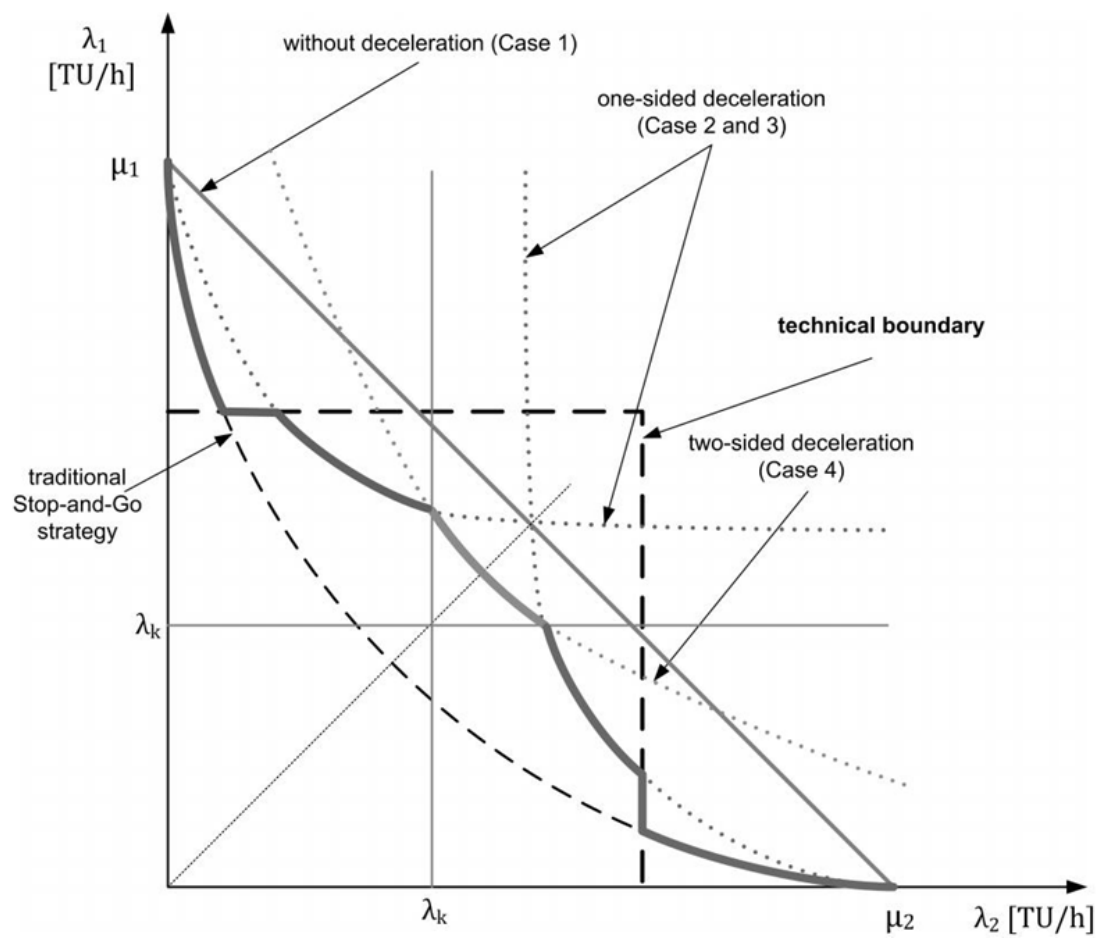

Illustration 8. Boundaries of the dynamic merge in the throughput diagram.

Because of the technical boundary of the dynamic prioritized merge it is not possible to operate in this mode close to the maximum throughputs $\mu_{1}$ and $\mu_{2}$. That means by reaching these boundary the units must be slow down till stop and accelerate again afterwards. So the system changes from the dynamic merge to a Stop-and-Go system. 


\section{Potentials For THE PRACTICE}

After the necessary equation systems are derived and qualitatively shown, it is to check which quantitative changes are expected. Therefore real equipment data was examined with an industrial partner and was compared to potentials determined by measuring and practice.

- Unit length

- Conveyor speed

- Maximum speed in the acceleration belt $v_{\max }=0.9\left[\mathrm{~m} \mathrm{~s}^{-1}\right]$

- Minimum speed in the deceleration belt $v_{\text {min }}=0.3\left[\mathrm{~m} \mathrm{~s}^{-1}\right]$

- Maximum acceleration of the units in Stop-and-Go systems $\quad a^{+}=1.8\left[\mathrm{~m} \mathrm{~s}^{-2}\right]$

- Maximum deceleration of the units in Stop-and-Go systems

- Steady portion of switching time

- Minimum unit distance

$$
\begin{array}{ll}
\mathrm{s} & =0.8[\mathrm{~m}] \\
v & =0.6\left[\mathrm{~m} \mathrm{~s}^{-1}\right]
\end{array}
$$

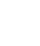$$
a^{-} \quad=1.8\left[\mathrm{~ms}^{-2}\right]
$$$$
\Delta s_{\min }=0.1[\mathrm{~m}]
$$$$
t_{f}=0.1[\mathrm{~s}]
$$

The solution for the traditional Stop-and-Go technology is used as a comparison (see (3)). The switching time $t_{0}$ of this traditional technology calculates itself after the following equation to:

$$
\mathrm{t}_{0}=\mathrm{t}_{\mathrm{f}}+\mathrm{t}_{\mathrm{a}^{-}}+\mathrm{t}_{\mathrm{a}^{+}}
$$

It includes the fixed switching time portion $t_{f}$, the deceleration time $t_{a}$ and the acceleration time $t_{a^{+}}$. The deceleration- and acceleration times can be calculated by knowledge of the maximum acceleration $a^{+}$, the maximum deceleration $a^{-}$and the conveyor speed $\mathrm{v}$ by the following equations:

$$
\begin{aligned}
\mathrm{t}_{\mathrm{a}^{-}}=\frac{\mathrm{v}}{\mathrm{a}^{-}}=\frac{0.6}{1.8} & =0.33 \mathrm{~s} \text { and } \mathrm{t}_{\mathrm{a}^{+}}=\frac{\mathrm{v}}{\mathrm{a}^{+}} \\
& =\frac{0.6}{1.8}=0.33 \mathrm{~s}
\end{aligned}
$$

The result is the switching time of the Stop-and-Go system:

$$
\begin{aligned}
\mathrm{t}_{0}=\mathrm{t}_{\mathrm{f}}+\mathrm{t}_{\mathrm{a}^{-}}+\mathrm{t}_{\mathrm{a}^{+}} & \\
& =0.1 \mathrm{~s}+0.33 \mathrm{~s}+0.33 \mathrm{~s} \\
& =0.76 \mathrm{~s}
\end{aligned}
$$

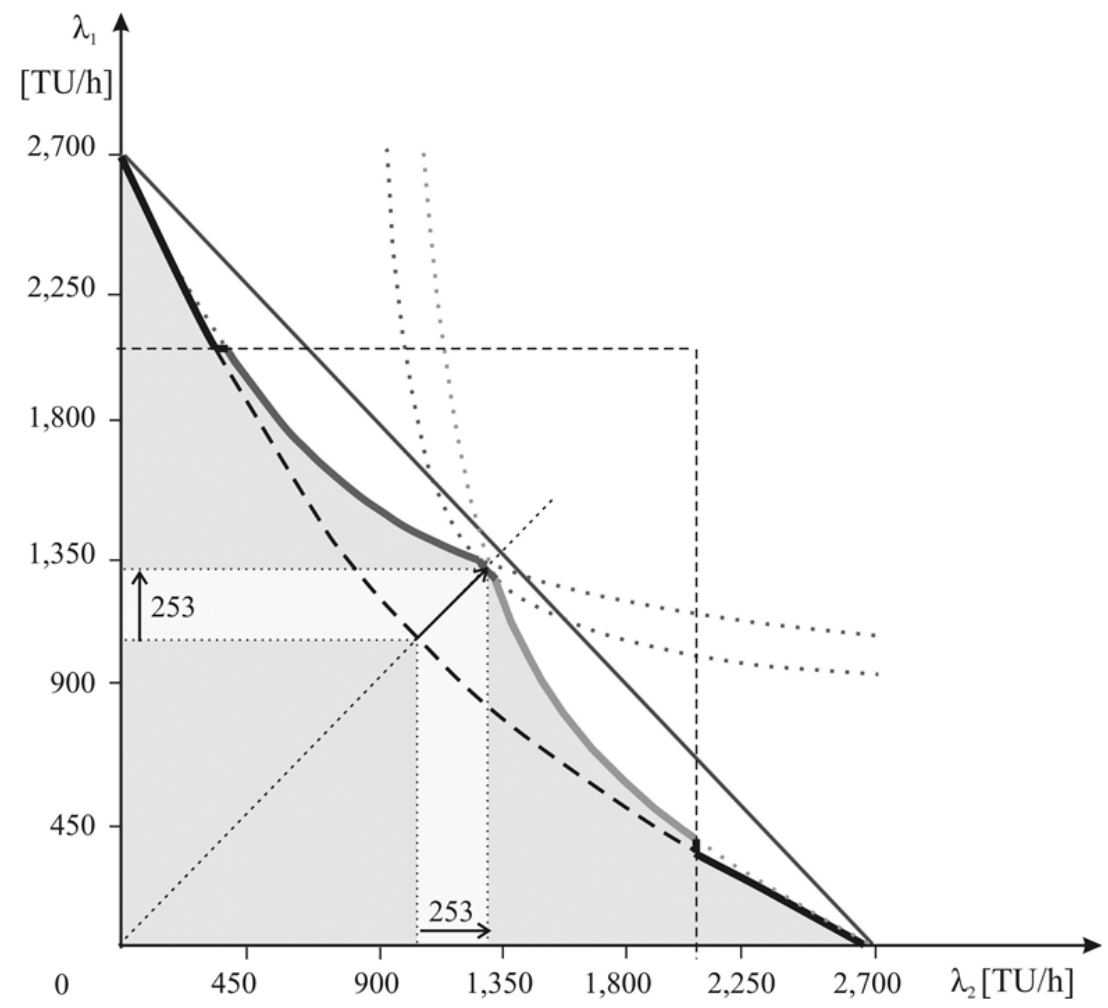

Illustration 9. Throughput diagram with real industrial data 
The boundary of the solution space is marked in the throughput diagram in illustration 9. The results of the unequations of Table 2 calculated with the real values are also inserted, so that potentials can be deduced directly from the diagram.

It is immediately observable that significant advantages of the dynamic merge can only exist in the direct surroundings of a symmetrical limiting performance $\lambda_{1} \approx \lambda_{2}$. The maximum advantage of the present parameter combination is an approx. $24 \%$ higher throughput (2,597 TU instead of 2,091 TU per hour) with the same partial flows, which quickly decreases below $10 \%$ with unequal partial flows. These results coincide with the measuring results and observations of the industrial partner. $24 \%$ seems not to be so much, but a look to Illustration 9 shows, that this value is very close to the characteristic-line without switching time. The maximum throuput would be 2,700 TU per hour.

Finally there is still one question. How much extent these maximum potentials by higher performances? Therefore the equations got new calculated with respectively single parameter changes.

Table 3. Scenarios

\begin{tabular}{|c|c|c|c|c|}
\hline no. & \multicolumn{2}{|c|}{ modification } & $\lambda_{\max }$ & $\begin{array}{c}\text { advantages compared } \\
\text { with the Stop-and-Go } \\
\text { area* } \lambda_{1} \approx \lambda_{2}\end{array}$ \\
\hline 1 & unit length $\mathrm{s}$ & $0.8 \mathrm{~m} \rightarrow 0.6 \mathrm{~m}$ & 3,420 & $31 \%$ \\
\hline 2 & conveyor speed $* * v^{*}$ & $0.6 \mathrm{~m} \mathrm{~s}^{-1} \rightarrow 1.0 \mathrm{~m} \mathrm{~s}^{-1}$ & $4,230 * *$ & $65 \%$ \\
\hline 3 & minimal unit distance $\Delta s_{\min }$ & $0.1 \mathrm{~m} \rightarrow 0.05 \mathrm{~m}$ & 2,645 & $26 \%$ \\
\hline 4 & combination of $1+2+3$ & & 5,820 & $95 \%$ \\
\hline 5 & combination of $1+2$ & & 5,633 & $89 \%$ \\
\hline 6 & combination of $1+3$ & & 3,510 & $35 \%$ \\
\hline
\end{tabular}

*In reference to the also moving Stop- and-Go curve by parameter changes

** Calculated with constant unit distance. In the reality it could be necessary to have larger distances.

The throughputs were detected in the operating state $\lambda_{1} \approx \lambda_{2}$ which always is located in the operating range. So they are valid in any case, also taking into account the technical boundary. The reason is that the enlargement of the speed or a reduction of the safety distance leads to a suitable enlargement of these natural boundaries.

For scenario 2 the speeds were raised. As expected, the potentials become here clearly bigger because stopping and acceleration needs more time. All together it can be said that in consideration of additional investments of approx. $10 \%$ by additional conveyor systems and adjustable drives in the merge, every use needs to be checked.

\section{Conclusion}

Compared to traditional Stop-and-Go systems, clearly higher throughput of a dynamic merge exists, especially with higher speeds and symmetrical partial flows. The throughput may increase to a maximum of $24 \%$ given usual speeds of $0.6 \mathrm{~m} / \mathrm{s}$. With higher speeds of $1 \mathrm{~m} / \mathrm{s}$ the throughput can increases up to $65 \%$. Therefore it can be said that an amount of work needs less time because of the higher throughput. However this efficiency profit must be probably confronted with additional energy consumption which is caused by control and frequency converter, as well as energy losses pursued by engines running beyond nominal power. These possible losses will be examined within the research project mentioned in the introduction to this article.

Positive effect of the dynamic merge remains the steadier and more harmonious process of the merge, which is caused by absence of stops. Material handling facilities with higher speeds can expect considerable increase of output.

\section{LITERATURE}

[JoW10] JodIn, Dirk; WoLfSCHLUCKNER, Andreas (2010): Merge problems with high speed sorters. In: Material Handling Institute (Hrsg.): Progress in Material Handling Re- 
search: 2010. USA : 2010, ISBN 978-1882780-16-7, S. 186 - 196.

[Jod10] Jodin, Dirk (2010): Dynamische Gutzusammenführung (Merge) auf Stetigförderern. Miskolc: Publiziert auf DVD, 2010.

[ArF07] ARNOLD, Dieter; FuRMANS, Kai (2005): Materialfluss in Logistiksystemen. Berlin [u.a.]: 5. erw. Aufl., Springer, 2007. ISBN 3-540-45659-7.

[Gud76a] GudEHUS, Timm (1976): Grenzleistungen bei absoluter Vorfahrt. In: Zeitschrift für Operations Research 20 (1976), S. B207B252, 1976.

[Gud76b] GUDEHUS, Timm (1976): Grenzleistungsgesetze für Verteiler- und Sammelelemente. In: Zeitschrift für Operations Research 20 (1976) S. B37-B61, 1976. 